\title{
Effect of theophylline on CD11b and L-selectin expression and density of eosinophils and neutrophils in vitro
}

\author{
F.M. Spoelstra, C. Berends, B. Dijkhuizen, J.G.R. de Monchy, H.F. Kauffman
}

\begin{abstract}
Effect of theophylline on CD11b and L-selectin expression and density of eosinophils and neutrophils in vitro. F.M. Spoelstra, C. Berends, B. Dijkhuizen, J.G.R. de Monchy, H.F. Kauffman. CERS Journals Ltd 1998.

ABSTRACT: The nonspecific phosphodiesterase inhibitor theophylline, widely used in asthma therapy, may cause a decrease in inflammatory responses of airways. In asthma, eosinophils migrate to the airway wall and become activated. Activated eosinophils are characterized by low cell density, as well as increased expression of CD11b and reduced expression of L-selectin, two adhesion molecules involved in transendothelial migration.

To study the anti-inflammatory effect of theophylline on granulocyte adhesion molecules in vitro, the platelet-activating factor (PAF)-induced density shift was determined by density centrifugation and the modulation of CD11b and L-selectin expression by flow cytometry on eosinophils and neutrophils in human whole blood.

A relatively high concentration of theophylline $\left(10^{-3} \mathrm{M}\right)$ inhibited the increase in the percentage of hypodense eosinophils and neutrophils in whole-blood samples after PAF stimulation in vitro. A more pharmacological concentration $\left(10^{-4} \mathrm{M}\right)$ inhibited the CD11b upregulation and L-selectin shedding induced by PAF $\left(10^{-7} \mathrm{M}\right)$ on both eosinophils and neutrophils. The effect of isoproterenol on the inhibitory effect of theophylline was mainly additive, but a small synergistic effect could not be excluded.

In conclusion theophylline can attenuate esinophil and neutrophil activation in vitro at the level of adhesion molecule expression and changes in cell density. This may have implications for transendothelial migration of these cells in asthma.
\end{abstract}

Eur Respir J 1998; 12: 585-591.

Dept of Allergology, University Hospital Groningen, Groningen, The Netherlands.

Correspondence: H.F. Kauffman

Laboratory of Allergology

Clinic for Internal Medicine

University Hospital Groningen

Hanzeplein 1

9713 GZ Groningen

The Netherlands

Fax: 310503121576

Keywords: Asthma

eosinophils

neutrophils

theophylline

Received: April 171997

Accepted after revision March 171998

Supported by a grant from 3M Pharma.
In asthma, eosinophils contribute to inflammation of the lung tissue and additional infiltration of eosinophils into the airway wall has been observed during the allergen-induced late asthmatic reaction (LAR) [1]. After infiltration, the eosinophils are activated and may release toxic granule components, superoxide radicals and leukotrienes, which may cause bronchoconstriction and epithelial damage $[2,3]$. Neutrophils may also play a role in the pathogenesis of asthma, since they have been observed in the lung tissue after allergen challenge and are found in greater numbers in the bronchoalveolar lavage fluid (BALF) of asthmatics than of nonatopic controls [4].

Adhesion of leukocytes to the endothelium is a primary event in the inflammatory response of the airways. In the process of adhesion to the vascular wall, which precedes transendothelial migration and infiltration into the lung tissue, L-selectin on granulocytes is the adhesion molecule responsible for rolling the cells along the endothelium [5]. Shedding of L-selectin is followed by firm adhesion mediated by $\beta$-integrins such as CR3 (CD11b/CD18), which bind to endothelial cellular adhesion molecules such as intracellular adhesion molecule (ICAM)-1 [6].

In vitro activation by endothelial products, such as platelet-activating factor (PAF), induces a rapid release of $\mathrm{CD} 11 \mathrm{~b}$ from intracellular compartments and an elevated CD11b membrane expression [7], while L-selectin is shed from the cell surface [8]. Furthermore, PAF induces a shift towards low cell density [9], which is an additional marker for activation $[10,11]$.

The drug theophylline has been used in asthma therapy for over $50 \mathrm{yrs}$, but the precise mechanisms responsible for its beneficial effects have not yet been elucidated [12, 13]. In high concentrations, theophylline acts as a bronchodilator, relaxing airway smooth muscle [14]. However, during therapy, plasma levels do not reach these concentrations. Evidence of anti-inflammatory effects of theophylline at therapeutic plasma levels $[15,16]\left(0.5 \times 10^{-4} \mathrm{M}\right.$, approximately equal to $\left.9 \mathrm{mg} \cdot \mathrm{L}^{-1}\right)$ has been obtained recently [15-17]. Theophylline significantly inhibits latephase eosinophil infiltration into the airways of sensitized and challenged guinea-pigs [18]. The number of eosinophils in the BALF of guinea-pigs after intratracheal administration of interleukin (IL)-5 was reduced by prior theophylline injection [19]. Moreover, in humans, low-dose theophylline can reduce the number of infiltrating and EG2positive eosinophils in the bronchial mucosa after allergen challenge [17]. In nocturnal asthma, theophylline decreases the number of neutrophils found in BALF [20].

The underlying mechanism for direct effects on inflammatory cells may be the phosphodiesterase (PDE)-inhibitory properties of theophylline, resulting in higher intracellular cyclic adenosine monophosphate (cAMP) levels, thereby 
inhibiting cell activation [14]. Theophylline is able to modulate the activation of inflammatory cells, such as the inhibition of the C5a-induced eosinophil cationic protein (ECP) and eosinophil-derived neutrotoxin (EDN) secretion by eosinophils, as well as the formation of reactive oxygen species [21]. A second mechanism for the inhibition of granulocyte activation is the modulation of adhesion molecule expression needed for attachment to the endothelium and subsequent transendothelial migration, resulting in cell recruitment into the airways. Recently, it was shown that rolipram, a specific PDE-inhibitor, is able to inhibit PAF-induced modulation of adhesion molecule expression on eosinophils and neutrophils [22].

In this study, the hypothesis that theophylline can inhibit the PAF-induced modulation of adhesion molecule expression on peripheral blood eosinophils and neutrophils in vitro was tested. CD11b and L-selectin expression and the PAF-induced shift towards low cell density were used as indicators of activation. Additionally, the study tested whether the $\beta$-agonist isoproterenol is able to act synergistically with theophylline in the inhibition of modulation of adhesion molecule expression induced by PAF. The effect of theophylline on granulocytes of healthy controls was also compared with the effect on granulocytes of asthmatic patients as well as asthmatic patients receiving corticosteroid treatment. All inhibition experiments were performed in whole-blood samples.

\section{Materials and methods}

\section{Study design}

To determine the influence of theophylline on the PAFinduced shift in densities of eosinophils and neutrophils, whole blood samples were incubated with theophylline or Hank's balanced salt solution (HBSS) (control) followed by incubation with PAF. These studies were performed with blood samples from healthy controls, asthmatics receiving no corticosteroid treatment (asthmatics CS-) and asthmatics receiving corticosteroid treatment (asthmatics CS+). Cells were monitored for density using density gradient centrifugation on isotonic Percoll and collection of cells from the interfaces. Percentages of hypodense neutrophils and eosinophils were calculated as described previously [10]. In addition, the effect of theophylline and/or isoproterenol on the PAF-induced modulation of CD11b and L-selectin expression on eosinophils and neutrophils in whole-blood samples was determined using flow cytometric analysis [23].

\section{Materials}

Anti-Leu15 (CD11b), anti-Leu8 (L-selectin) and goat anti-mouse immunoglobulin (Ig) fluorescein isothiocyanate (FITC) (GAM-FITC) were purchased from Becton Dickinson (San Jose, CA, USA). Mouse isotype control antibody (IgG2a) was obtained from the Central Laboratory of the Netherlands Red Cross Blood Transfusion Service (Amsterdam, The Netherlands). Polyvinyl pyrrolidonecoated silica gel (Percoll) was purchased from Pharmacia LKB (Uppsala, Sweden). $\beta$-acetyl- $\tau$ - $O$-hexadecyl-L- $\alpha$-phosphatidylcholine (PAF) and (-)isoproterenol hydrochloride were obtained from Sigma Chemical Co. (St. Louis, MO, USA). Bovine albumin solution $200 \mathrm{~g} \cdot \mathrm{mL}^{-1}$ containing 1 $\mathrm{g} \cdot \mathrm{L}^{-1}$ sodium azide (Boseral 20T) was obtained from Organon Teknika B.V. (Boxtel, The Netherlands). HBSS was purchased from Gibco BRL (Grand Island, NY, USA). Theophylline was kindly provided by $3 \mathrm{M}$ Pharma.

\section{Subjects}

Twenty four subjects were included in this study. Twelve healthy people formed the control group. Subjects were included if they were 18-40 yrs of age, nonsmokers, had no history of allergy or asthma, had a forced expiratory volume in one second $(\mathrm{FEV} 1)>90 \%$ predicted, were specifically IgE-negative for a panel of aeroallergens (housedust mite (HDM), grass and tree pollen, cat and dog) as determined by fluorescent enzyme immunoassay (FEIA) and had used no corticosteroids or nonsteroidal antiinflammatory drugs (NSAID) for at least 7 days.

Six asthmatics CS- and 6 asthmatics CS+ were included. They were 18-40 yrs of age, nonsmokers, had a diagnosis of mild asthma with a forced expiratory volume in one second (FEV1) Š70\% pred, a provocative concentration of histamine causing a $20 \%$ fall in FEV1 (PC20) ð8 $\mathrm{mg} \cdot \mathrm{mL}^{-1}$, were sensitized (serum IgE determined by FEIA or skin-prick test) to at least one of the following aeroallergens: HDM, grass or tree pollen, cat or dog, and had used no $\beta$-adrenoceptor agonists, anticholinergics, cromoglycate or nedocromil within the last $8 \mathrm{~h}$, no antihistaminic drugs within the last 3 days, no NSAID for at least 7 days, no theophylline for 4 weeks and no corticosteroids in the asthmatics CS- group for at least 4 weeks.

\section{Density of eosinophils and neutrophils}

Venous blood (4 mL) anticoagulated with ethylenediaminetetraacetic acid (EDTA) was preincubated with $0.9 \%$ saline or $10^{-3} \mathrm{M}$ theophylline for $15 \mathrm{~min}$ at $37^{\circ} \mathrm{C}$. Subsequently, the blood was incubated with $10^{-7} \mathrm{M}$ PAF for 15 min at $37^{\circ} \mathrm{C}$. Control values were obtained from blood samples without incubation with theophylline or PAF. After the incubations, an equal volume of $0.9 \%$ saline was mixed with the blood samples before they were layered onto a discontinuous isotonic Percoll gradient consisting of the following densities: 1.070, 1.075, 1.080, 1.085 and $1.090 \mathrm{~g} \cdot \mathrm{mL}^{-1}$. After centrifugation at $1,000 \times \mathrm{g}$ and $20^{\circ} \mathrm{C}$ for $20 \mathrm{~min}$, cells were collected from the interfaces and the bottoms of the tubes and washed in phosphate-buffered saline (PBS). Erythrocytes were lysed in cold ammonium chloride $\left(155 \mathrm{mM} \mathrm{NH}_{4} \mathrm{Cl}, 10 \mathrm{mM} \mathrm{KHCO}, 0.1 \mathrm{mM}\right.$ $\mathrm{Na}_{2}$ EDTA) for $5 \mathrm{~min}$ at $4^{\circ} \mathrm{C}$. The cells were washed and reconstituted in PBS. Leukocytes sampled at the different interfaces were counted using a Coulter Counter (model S880; Coulter Electronics, Hialeah, FL, USA). Differential cell counts were made using cytocentrifuge slides stained with May-Grünwald Giemsa. Percentages of total eosinophils and neutrophils recovered at each density interface were calculated. Granulocytes collected from densities be-low $1.085 \mathrm{~g} \cdot \mathrm{mL}^{-1}$ were considered to be hypodense. This density is based on results from healthy controls, where up to $10 \%$ of granulocytes are permitted to be hypodense [10]. 
Flow cytometric analysis of CD1 $1 b$ and L-selectin

Venous blood was collected in tubes containing EDTA and centrifuged $(590 \times \mathrm{g}, 10 \mathrm{~min})$. The pellet was washed with PBS and the original volume was restored with PBS. Cell suspensions of $100 \mu \mathrm{L}$ were preincubated with $10^{-5} \mathrm{M}$ or $10^{-4} \mathrm{M}$ theophylline or HBSS for $15 \mathrm{~min}$ at $37^{\circ} \mathrm{C}$, followed by incubation with $10^{-7} \mathrm{M}$ PAF with or without $0.5 \times 10^{-3} \mathrm{M}$ isoproterenol for $15 \mathrm{~min}$ at $37^{\circ} \mathrm{C}$. In preliminary experiments, incubations with theophylline at concentrations of $10^{-3} \mathrm{M}$ to $10^{-6} \mathrm{M}$ were performed in order to determine optimal concentrations. Control samples were incubated with HBSS only. Immunofluorescent staining of the cells was performed as described by BERENDS et al. [22]. Cell fluorescence was measured with a FACStar (Becton Dickinson). Before measurement, the fluorescence-activated cell sorter (FACS) was calibrated using standardized fluorescent Quick Cells 3 beads (Flow Cytometry Standards Co., San Juan, Puerto Rico). Discrimination between eosinophils and neutrophils was achieved by a technique using a polarizing filter [24]. Eosinophils show a more depolarized orthogonal light scattering than neutrophils [23]. In earlier experiments no contaminating cells could be observed in these two populations [23].

Expression of L-selectin and CD11b was calculated as mean fluorescence intensity (MFI) in linear values, using the Lysis program (Becton Dickinson). Fluorescence due to control antibody was subtracted from these values. In order to compare CD11b and L-selectin expressions after treatment with theophylline and/or isoproterenol, the expressions were calculated as a percentage of the response induced by PAF alone.

\section{Statistical analysis}

Normally distributed data were presented as mean \pm SD. The differences in CD11b, L-selectin and percentages of hypodense cells induced by theophylline and isoproterenol were normally distributed according to the Kolmogorov-Smirnov goodness-of-fit test and were evaluated by the Student's t-test for paired data. For investigating differences between the three subject groups, statistical analysis was performed using the Mann-Whitney U-test and data were presented as median percentages (minimum-maximum).

\section{Results}

\section{Influence of theophylline on the PAF-induced density shift}

Since the activating effects of PAF on eosinophils and neutrophils were similar in all groups, the overall effect was studied of theophylline on cell activation in the total group (asthmatics plus controls). Figures 1 and 2 show that PAF induced a substantial shift in the density of eosinophils and neutrophils towards lower densities. Addition of $10^{-3} \mathrm{M}$ theophylline before incubation with PAF showed inhibition of the PAF-induced shift for both cell types. The percentage of low-density eosinophils in nontreated whole-blood samples was $1.7 \pm 2.8 \%$, and this percentage increased significantly $(\mathrm{p}<0.05)$ to $32.8 \pm 19.7 \%$

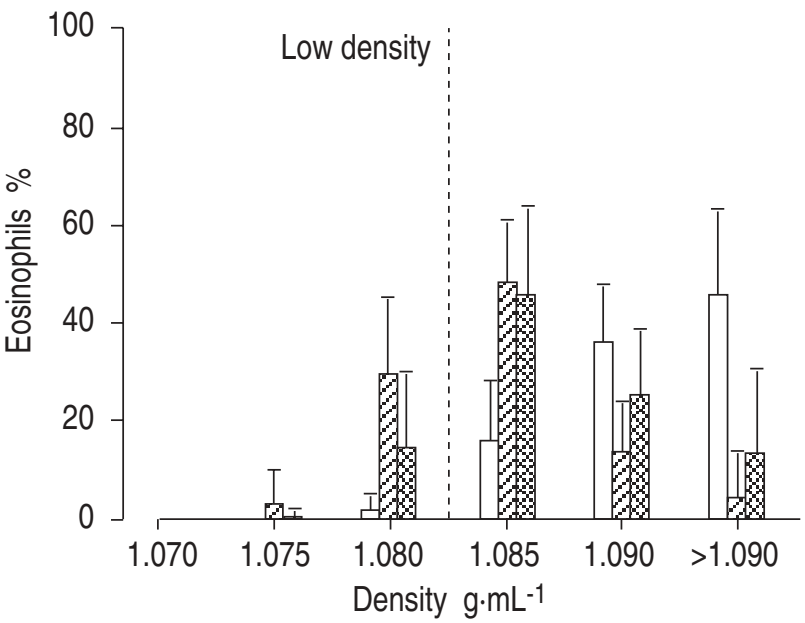

Fig. 1. - Effect of theophylline on the density of eosinophils $(n=24)$. Whole-blood samples were centrifuged on Percoll density gradients directly ( $\square$ ), after stimulation with platelet-activating factor ((PAF), 10-7 M) ( $\nabla$ ) or after incubation with $10^{-3} \mathrm{M}$ theophylline prior to stimulation with PAF ( $\%$ ). Results are presented as mean percentage \pm SD of total eosinophils recovered. Eosinophils with a density of $\partial 1.080 \mathrm{~g} \cdot \mathrm{mL}^{-1}$ were defined as hypodense (- - - ).

after incubation with PAF. Preincubation with theophylline and sub-sequent stimulation with PAF significantly inhibited the generation of low-density eosinophils $(14.7 \pm 15.8 \%$, $\mathrm{p}<0.05)$ compared with the percentage of PAF-induced low-density cells.

Neutrophils showed higher percentages of low-density cells after incubation with PAF compared with eosinophils. A similar inhibition was observed with theophylline. Direct separation of whole-blood cells yielded $2.7 \pm 2.6 \%$ of low-density neutrophils and this percentage increased significantly $(\mathrm{p}<0.05)$ after incubation with PAF to $90.5 \pm$ $8.3 \%$. When PAF and theophylline were both present, lowdensity neutrophils increased to $72.0 \pm 19.4 \%$ of the total neutrophil counts. This was a significantly smaller percentage than after incubation with PAF alone $(\mathrm{p}<0.05)$.

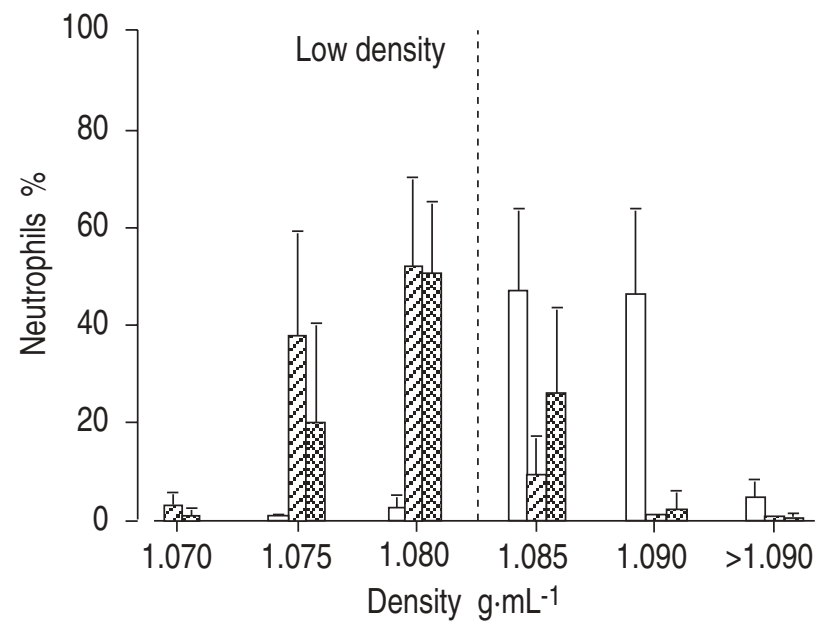

Fig. 2. - Effect of theophylline on the density of neutrophils $(n=24)$. Whole-blood samples were centrifuged on Percoll density gradients directly ( $\square$ ), after stimulation with platelet-activating factor ((PAF) $10^{-7}$ M) ( $Q$ ) or after incubation with $10^{-3} \mathrm{M}$ theophylline prior to stimulation with PAF $(\propto)$ ). Results are presented as mean percentage \pm SD of total neutrophils recovered. Neutrophils with a density of $\partial 1.080 \mathrm{~g} \cdot \mathrm{mL}^{-1}$ were defined as hypodense (- - -). 
Table 1. - Effect of theophylline on the platelet-activating factor (PAF)-induced shift in density of neutrophils and eosinophils

\begin{tabular}{|c|c|c|c|c|c|c|c|c|}
\hline \multirow[b]{2}{*}{ Group } & \multicolumn{3}{|c|}{ Low-density neutrophils \% } & \multicolumn{5}{|c|}{ Low-density eosinophils \% } \\
\hline & A & B & $\mathrm{C}$ & A & & B & & $\mathrm{C}$ \\
\hline$\overline{\mathrm{HC}}$ & $1(0.5-8)$ & $95(88-99)$ & $82(43-99)$ & $1(0-8)$ & 36 & $(0-79)$ & 12 & $(0-57)$ \\
\hline CS- & $3(0-9)$ & $88(78-97)^{*}$ & 73 (63-97) & $2(0-12)$ & 25 & $(14-69)$ & 10 & $(3-53)$ \\
\hline $\mathrm{CS}+$ & $2(1-4)$ & 89 (68-99) & $60(27-97)$ & $0(0-2)$ & 19 & $(4-55)$ & 6 & $(0-21)$ \\
\hline
\end{tabular}

Results represent median percentages (minimum-maximum). Low-density is defined as $01.080 \mathrm{~g} \cdot \mathrm{mL}^{-1}$. HC: healthy controls $(n=12)$; CS-: asthmatic patients without corticosteroid treatment $(n=6)$; CS+: asthmatic patients using corticosteroids $(n=6)$. A: directly separated whole-blood samples; B: whole-blood samples incubated with $10^{-7} \mathrm{M}$ PAF for $15 \mathrm{~min}$ at $37^{\circ} \mathrm{C}$; C: whole-blood samples preincubated with $10^{-3} \mathrm{M}$ theophylline for $15 \mathrm{~min}$ at $37^{\circ} \mathrm{C}$ before incubation with PAF. *: $\mathrm{p}<0.05$ compared with healthy controls, Mann-Whitney U-test.

A lower concentration of theophylline $\left(10^{-4} \mathrm{M}\right)$, in the presence or absence of the $\beta$-adrenergic stimulant isoproterenol $\left(0.5 \times 10^{-8} \mathrm{M}\right)$, had no effect on the PAF-induced shift towards a lower density of eosinophils and neutrophils (data not shown).

In table 1 , the effect of PAF and theophylline is shown for the different subject groups (controls, asthmatics CSand asthmatics $\mathrm{CS}+$ ). The inhibitory effect of $10^{-3} \mathrm{M}$ theophylline on the PAF-induced increase in low-density cells (ð1.080 $\left.\mathrm{g} \cdot \mathrm{mL}^{-1}\right)$ did not reach significance between the three subject groups. A significantly smaller number of low-density neutrophils were found in the asthmatics CSgroup after activation with PAF compared with the control group (table 1).

Influence of theophylline on modulation of CD11b and $L_{-}$ selectin expression

CD11b expression increased and L-selectin expression decreased significantly on eosinophils and neutrophils after stimulation with PAF compared with HBSS control values (table 2). Theophylline, in the presence or absence of isoproterenol, affected the expression of CD11b and Lselectin on eosinophils and neutrophils after stimulation with PAF. As in the density study, analysis of the effect of theophylline and isoproterenol was performed on the combined subject groups. Isoproterenol alone inhibited the PAF-induced upregulation of CD11b on eosinophils and neutrophils (fig. 3) and downregulation of L-selectin on neutrophils (fig. 4) $(\mathrm{p}<0.05)$. Theophylline $\left(10^{-4} \mathrm{M}\right)$ had a significant inhibitory effect on the PAF-induced modulation of CD11b and L-selectin expression on both cell types. Expression of CD11b was reduced to $68.8 \pm 7.5 \%$ and $82.4 \pm 4.9 \%$ of the values obtained from PAF alone on eosinophils and neutrophils, respectively (fig. 3). A concentration of $10^{-4} \mathrm{M}$ theophylline significantly inhibited

Table 2. - Effect of platelet-activating factor (PAF) on CD11b and L-selectin expression on eosinophils and neutrophils

\begin{tabular}{lrrrrr}
\hline & \multicolumn{2}{c}{ Eosinophils } & & \multicolumn{2}{c}{ Neutrophils } \\
\cline { 2 - 3 } \cline { 5 - 6 } & HBSS & PAF & & HBSS & PAF \\
\hline CD11b & $116 \pm 44$ & $310 \pm 76^{* * *}$ & & $96 \pm 74$ & $672 \pm 179^{* * *}$ \\
L-Selectin & $94 \pm 51$ & $43 \pm 31^{* * *}$ & & $281 \pm 142$ & $103 \pm 81^{* * *}$
\end{tabular}

Results represent mean percentages $\pm S D(n=24)$ in mean fluorescence intensity values. HBSS: Hank's balanced salt solution. $* * *: \mathrm{p}<0.001$ compared with HBSS, Student's paired t-test. downregulation of L-selectin on both eosinophils and neutrophils $(67.1 \pm 17.9 \%$ and $80.0 \pm 8.3 \%$ of the values obtained by PAF alone, respectively) (fig. 4). The combination of theophylline $\left(10^{-4} \mathrm{M}\right)$ and isoproterenol $\left(0.5 \times 10^{-8} \mathrm{M}\right)$ showed even stronger inhibitory effects, which were significantly different from the effects of isoproterenol alone. However, no inhibitory effects of $10^{-5} \mathrm{M}$ theophylline were found. The combination of $10^{-5} \mathrm{M}$ theophylline and isoproterenol significantly inhibited the PAF-induced modulation on eosinophils (CD11b: 69.5 $\pm 7.1 \%$; L-selectin: $57.0 \pm 10.5 \%$ ) and neutrophils (CD11b: $72.9 \pm 4.9 \%$; L-selectin: $64.3 \pm 5.6 \%$ ), but no significant difference was found compared with incubations with isoproterenol alone. The effects of isoproterenol and theophylline on modulation of $\mathrm{CD} 11 \mathrm{~b}$ and L-selectin expression were mainly additive, except for $10^{-5} \mathrm{M}$ theophylline on L-selectin shedding of neutrophils, where it was found to be synergistic, giving significantly $(\mathrm{p}=0.04)$ higher percentages of inhibition in the presence of isoproterenol and theophylline than with either substance alone.

Theophylline had no effect on the expression of CD11b and L-selectin on resting granulocytes from samples incubated with HBSS (not shown).

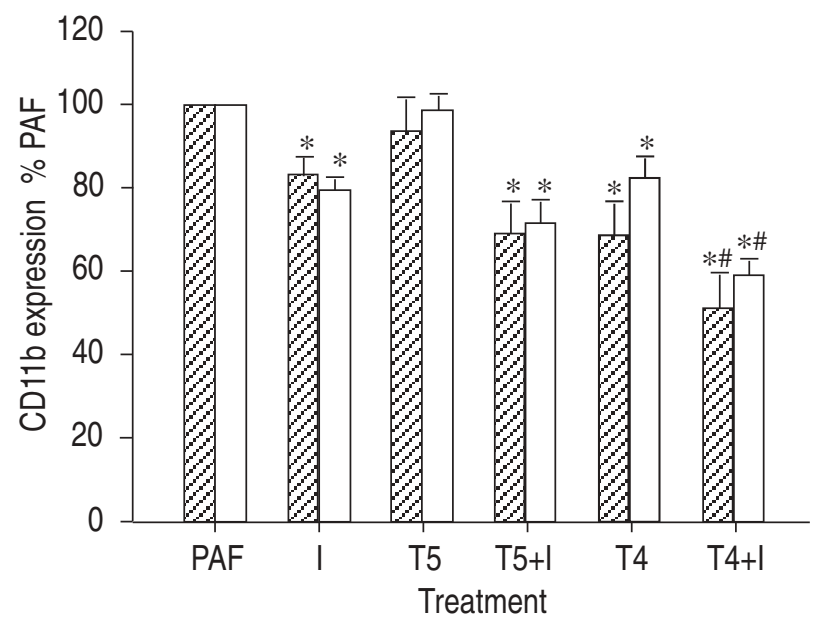

Fig. 3. - Effects of theophylline at $10^{-4} \mathrm{M}$ (T4) and $10^{-5} \mathrm{M}$ (T5), and $0.5 \times 10^{-8} \mathrm{M}$ isoproterenol (I) or both on the platelet-activating factor (PAF)-induced CD11b expression of eosinophils ( $Z$ ) and neutrophils ( $\square$ ). Incubation with T4/T5 and/or I was performed in whole blood prior to PAF $\left(10^{-7} \mathrm{M}\right)$. Results are presented as mean percentages \pm SD response compared to stimulation with PAF alone (\% PAF) $(n=24)$. The effect of theophylline or isoproterenol was significantly different $(\mathrm{p}<$ 0.05 ) from the effect of PAF alone (*) and from the effect of PAF and isoproterenol (\#). 


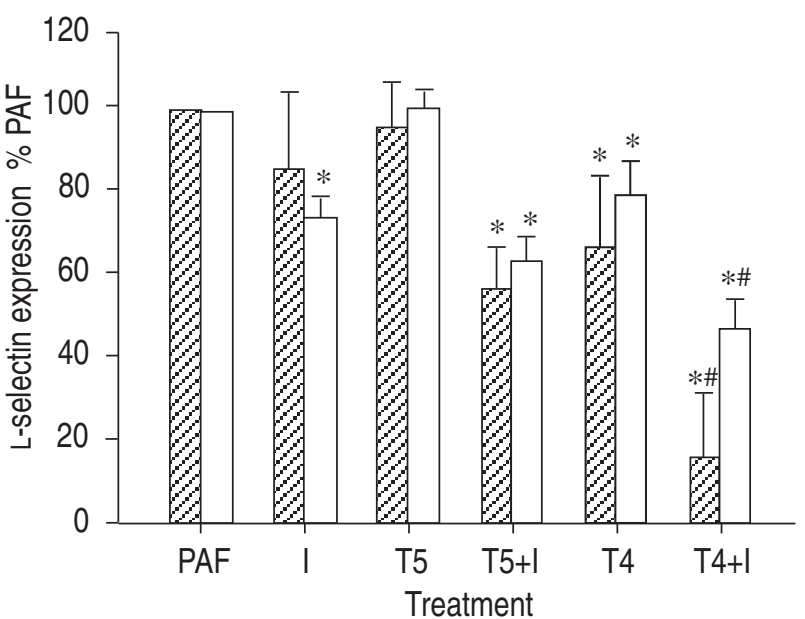

Fig. 4. - Effects of theophylline at $10^{-4} \mathrm{M}$ (T4) and $10^{-5} \mathrm{M}$ (T5), and $0.5 \times 10^{-8} \mathrm{M}$ isoproterenol (I) or both on platelet-activating factor (PAF)induced L-selectin downregulation on eosinophils ( 2$)$ ) and neutrophils $\square$ ). Incubation with T4/T5 and/or I was performed in whole blood prior to PAF $\left(10^{-7} \mathrm{M}\right)$. Results are presented as mean percentages \pm SD compared to stimulation with PAF alone (\% PAF) $(n=24)$. The effect of theophylline or isoproterenol was significantly different $(\mathrm{p}<0.05)$ from the effect of PAF alone $(*)$ and from the effect of PAF and isoproterenol (\#).

The inhibition of CD11b upregulation on eosinophils after incubation with $10^{-4} \mathrm{M}$ theophylline was stronger in the asthmatics $\mathrm{CS}+$ than in the control group (table 3). Incubation with $10^{-4} \mathrm{M}$ theophylline plus isoproterenol had a stronger inhibitory effect on CD11b upregulation on eosinophils from the asthmatics CS- group than from the control group (table 3). In contrast to the upregulation of CD11b, L-selectin downregulation on eosinophils induced by PAF was inhibited to a lesser extent in asthmatics CS+ than in healthy controls in the presence of theophylline $10^{-4}$ $\mathrm{M}$ in combination with isoproterenol (table 4). However, none of the differences between CD11b and L-selectin expression, after theophylline incubation, on neutrophils of the separate subject groups reached significance (not shown).

\section{Discussion}

In this study, theophylline $\left(10^{-3} \mathrm{M}\right)$ was demonstrated to have an inhibitory effect on the PAF-induced density shift of eosinophils and neutrophils in whole-blood samples. Furthermore, it was shown that in a near-therapeutic concentration $\left(10^{-4} \mathrm{M}\right)$, theophylline significantly inhibited the PAF-induced modulation of CD11b and L-selectin expression on eosinophils and neutrophils in vitro. Incubation with theophylline was performed in whole-blood samples, thereby keeping close to the in vivo situation and preventing possible activation of granulocytes by isolation procedures [10]. Isoproterenol is able to exert an additive inhibitory effect on the modulation of CD11b and L-selectin expression in combination with theophylline. A weak synergistic effect was also observed. There were no significant differences between healthy controls and asthmatic patients in the inhibitory effect of theophylline on the PAF-induced density shift in eosinophil and neutrophil populations, or in the CD11b and L-selectin expression on neutrophils. In contrast, differences were found between the subject groups in eosinophil CD11b and L-selectin expression after theophylline incubation.

Theophylline, at a concentration of $10^{-3} \mathrm{M}$, showed a reduction in the PAF-induced shift in cell density of eosinophils and neutrophils. However, no complete inhibition of the generation of hypodense eosinophils and neutrophils was observed $(64.6 \pm 30.4 \%$ and $21.8 \pm 17.8 \%$ inhibition, respectively). As the concentration of $10^{-3} \mathrm{M}$ theophylline will not be achieved in vivo, this observation is interesting in terms of the possible mechanisms involved, but is not therapeutically relevant.

The inhibitory effect of theophylline observed on the PAF-induced density shift seems to be more pronounced in eosinophils than in neutrophils, suggesting that eosinophils may be more susceptible to the action of theophylline in this system.

Neutrophils, after PAF treatment, showed significantly smaller low-density percentages in the asthmatics CS-group

Table 3. - Effect of theophylline on platelet-activating factor (PAF)-induced CD11b upregulation on eosinophils

\begin{tabular}{llrllll}
\hline & \multicolumn{5}{c}{ CD11b expression on eosinophils (\% of PAF response (100\%)) } \\
\cline { 2 - 6 } Group & \multicolumn{1}{c}{1} & \multicolumn{6}{c}{2} & 3 & \multicolumn{1}{c}{4} & 5 \\
\hline HC & $87(58-118)$ & $97(25-193)$ & $83(-11-103)$ & $84(42-102)$ & $62(41-91)$ \\
CS- & $76(49-135)$ & $108(81-116)$ & $67(58-104)$ & $77(50-98)$ & $49(4-60)^{*}$ \\
CS+ & $73(56-98)$ & $83(-29-126)$ & $69(-16-132)$ & $59(-84-78)^{*}$ & $48(-113-83)$ \\
\hline
\end{tabular}

Results represent median percentages (minimum-maximum). HC: healthy controls $(\mathrm{n}=12)$; CS-: asthmatic patients without corticosteroid treatment $(n=6)$; CS+: asthmatic patients using corticosteroids $(n=6)$. All samples were incubated with $10^{-7} \mathrm{M}$ PAF for 15 min at $37^{\circ} \mathrm{C}$ and additionally with 1 ) $0.5 \times 10^{-8} \mathrm{M}$ isoproterenol; 2) $10^{-5} \mathrm{M}$ theophylline; 3) $10^{-5} \mathrm{M}$ theophylline and $0.5 \times 10^{-8} \mathrm{M}$ isoproterenol; 4) $10^{-4} \mathrm{M}$ theophylline; 5) $10^{-4} \mathrm{M}$ theophylline and $0.5 \times 10^{-8} \mathrm{M}$ isoproterenol. *: p<0.05 compared with healthy controls, Mann-Whitney U-test.

Table 4. - Effect of theophylline on platelet-activating factor (PAF)-induced L-Selectin shedding on eosinophils

\begin{tabular}{|c|c|c|c|c|c|}
\hline \multirow[b]{2}{*}{ Group } & \multicolumn{5}{|c|}{ L-Selectin expression on eosinophils (\% of PAF response (100\%)) } \\
\hline & 1 & 2 & 3 & 4 & 5 \\
\hline$\overline{\mathrm{HC}}$ & $87(58-118)$ & $108(-98-204)$ & $69(-79-118)$ & $78(-215-174)$ & $24(-192-58)$ \\
\hline CS- & $76(40-93)$ & $102(87-118)$ & $52(38-75)$ & $102(31-118)$ & $13(-16-101)$ \\
\hline $\mathrm{CS}+$ & $89(25-296)$ & $114(71-174)$ & $85(25-176)$ & $88(57-161)$ & $62(46-135)^{*}$ \\
\hline
\end{tabular}

Results represent median percentages (minimum-maximum). HC: healthy controls $(n=12)$; CS-: asthmatic patients without corticosteroid treatment $(n=6)$; $C S+$ : asthmatic patients using corticosteroids $(n=6)$. All sample were incubated with $10^{-7} \mathrm{M}$ PAF for 15 min at $37^{\circ} \mathrm{C}$ and additionally with 1) $0.5 \times 10^{-8} \mathrm{M}$ isoproterenol; 2) $10^{-5} \mathrm{M}$ theophylline; 3) $10^{-5} \mathrm{M}$ theophylline and $0.5 \times 10^{-8} \mathrm{M}$ isoproterenol; 4) $10^{-4} \mathrm{M}$ theophylline; 5) $10^{-4} \mathrm{M}$ theophylline and $0.5 \times 10^{-8} \mathrm{M}$ isoproterenol. *: p<0.05 compared with healthy controls, Mann-Whitney U-test. 
than in the group of healthy controls. Although there is no direct explanation for this effect, the lower reactivity of neutrophils to PAF is suggestive of a desensitization in vivo.

At a therapeutically more relevant concentration $\left(10^{-4}\right.$ $\mathrm{M})$, theophylline inhibited the PAF-induced upregulation of CD11b and downregulation of L-selectin. This observation is consistent with the finding that another nonselective PDE inhibitor, pentoxifylline, is able to inhibit neutrophil CD11b expression [25] and is also consistent with the recent findings that rolipram, a type IV inhibitor of PDE, inhibits CD11b expression and L-selectin shedding from both eosinophils and neutrophils [22]. SaGara et al. [26] found an inhibition of the PAF-induced upregulation on isolated eosinophils by $10^{-4} \mathrm{M}$ and $10^{-5} \mathrm{M}$ theophylline. No significant inhibition on CD11b upregulation by $10^{-5} \mathrm{M}$ was found in the present study. This difference can be explained by the fact that whole-blood samples and a smaller PAF concentration were used in the current experiments. However, potentiation of eosinophil superoxide formation by low concentrations $\left(10^{-6}\right.$ and $\left.10^{-5} \mathrm{M}\right)$ of theophylline on isolated eosinophils has also been described. This effect is possibly due to an antagonistic effect of theophylline on adenosine receptors, while at higher concentrations the PDE inhibitory effects predominate [27]. No potentiation of activation was found with low concentrations of theophylline in the present study. The different effects of theophylline in this earlier study from those in the present study may be due to differences in the activation response or a difference in the cell suspensions used. Under in vivo conditions, the effects of theophylline may be enhanced by physiologically relevant $\beta$-agonists, such as adrenaline.

Therefore, the effect of theophylline was studied in the presence and absence of the $\beta$-agonist isoproterenol. It was shown that isoproterenol has an attenuating effect on the PAF-induced modulation of adhesion molecule expression on eosinophils and neutrophils. In combination with theophylline, the main effect was not synergistic, but additive. Only the combination of $10^{-5} \mathrm{M}$ theophylline and isoproterenol revealed a slight synergistic effect on the inhibition of L-selectin shedding from neutrophils. In comparable in vitro studies with PDE inhibitors and isoproterenol, synergistic effects have been observed on oxygen consumption [28]. The discrepancy with the current results could be due to differences in the regulation of the variables studied or to differences in the incubation procedures; in our study, theophylline was used in the preincubation of the cells, while isoproterenol was administered at the same time as the activating agent, PAF.

Differences in the capacity of theophylline for inhibiting PAF-induced changes in adhesion molecule expression between the three subject groups can be expected. It has recently been shown that atopic individuals have a higher PDE-activity in their inflammatory cells [14, 29, 30]. Therefore, cells from asthmatic patients might be more susceptible to the PDE-inhibitory capacity of theophylline. In this study, the combination of $10^{-4} \mathrm{M}$ theophylline and isoproterenol showed a greater inhibition of the CD11b upregulation on eosinophils from the asthmatics CS- group than from the control group. In the asthmatics CS+ group, similar differences were seen for $10^{-4} \mathrm{M}$ theophylline alone. These effects may be influenced by a higher PDE activity in eosinophils from asthmatic pat- ients. Furthermore, it has been suggested that steroids can act synergistically with PDE-inhibitors by virtue of their anti-inflammatory properties [12]. If this were true, theophylline would be expected to exert the greatest effect on cells of the asthmatics CS+ group. However, an opposite trend was seen in L-selectin shedding from eosinophils; there was a smaller inhibition in the asthmatics CS+ group when eosinophils were incubated with theophylline and isoproterenol. This difference in inhibition of CD11b and L-selectin modulation may be explained by different mechanisms underlying the expression of both adhesion molecules. However, lack of sufficient knowledge on the mechanism of CD11b upregulation and L-selectin shedding impedes an explanation of the opposite effects seen with theophylline in asthmatics CS+ versus healthy controls.

In summary, this study has shown that theophylline, in near therapeutically relevant concentrations, can rapidly attenuate eosinophil and neutrophil activation by inhibiting CD11b upregulation and L-selectin downregulation in vitro. This may have implications for the adhesion to endothelium and transendothelial migration of granulocytes in inflammatory tissues in patients with asthma.

Acknowledgements: The authors thank G.J. Jonker for selecting the asthmatic patients included in this study and A.E.J. Dubois for critically reading this manuscript.

\section{References}

1. de Monchy JGR, Kauffman HF, Venge P, et al. Bronchoalveolar eosinophilia during allergen-induced late asthmatic reactions. Am Rev Respir Dis 1985; 131: 373-376.

2. Gleich GJ, Flavahan NA, Fujisawa T, Vanhoutte PM. The eosinophil as a mediator of damage to respiratory epithelium: a model for bronchial hyperreactivity. J Allergy Clin Immunol 1988; 81: 776-781.

3. Venge P, Dahl R, Fredens K, Peterson CG. Epithelial injury by human eosinophils. Am Rev Respir Dis 1988; 138: S54-S57.

4. Bernstein IL. Asthma pathogenesis and the peripatetic polymorphonuclear granulocyte. Chest 1996; 110: 11381139.

5. Knol EF, Tackey F, Tedder TF, et al. Comparison of human eosinophil and neutrophil adhesion to endothelial cells under nonstatic conditions. Role of L-selectin. $J$ Immunol 1994; 153: 2161-2167.

6. Diamond MS, Staunton DE, de Fougerolles AR, et al. ICAM-1 (CD54): a counter-receptor for Mac-1 (CD11b/CD18). J Cell Biol 1990; 111: 3129-3139.

7. O'Shea JJ, Brown EJ, Seligmann BE, Metcalf JA, Frank $\mathrm{MM}$, Gallin Jl. Evidence for distinct intracellular pools of receptors for $\mathrm{C} 3 \mathrm{~b}$ and $\mathrm{C} 3 \mathrm{bi}$ in human neutrophils. $J$ Immunol 1985; 134: 2580-2587.

8. Neeley SP, Hamann KJ, White SR, Baranowski SL, Burch RA, Leff AR. Selective regulation of expression of surface adhesion molecules Mac-1, L-selectin, and VLA-4 on human eosinophils and neutrophils. Am J Respir Cell Mol Biol 1993; 8: 633-639.

9. Kloprogge E, de Leeuw AJ, de Monchy JGR, Kauffman HF. Hypodense eosinophilic granulocytes in normal individuals and patients with asthma: generation of hypodense cell populations in vitro. J Allergy Clin Immunol 1989; 83: 393-400. 
10. Berends C, Dijkhuizen B, de Monchy JGR, Gerritsen J, Kauffman HF. Induction of low density and up-regulation of CD11b expression of neutrophils and eosinophils by dextran sedimentation and centrifugation. J Immunol Methods 1994; 167: 183-193.

11. Winqvist I, Olofson T, Olsson I, Persson AM, Hallberg T. Altered density, metabolism and surface receptors of eosinophils in eosinophilia. Immunology 1982; 47: 531-539.

12. Barnes PJ, Pauwels RA. Theophylline in the management of asthma: time for a reappraisal? Eur Respir J 1994; 7 : 579-591

13. Magnussen H. Historical perspectives of theophylline treatment. Eur Respir Rev 1996; 6: 70-73.

14. Barnes PJ. Cyclic nucleotides and phosphodiesterases and airway function. Eur Respir J 1995; 8: 457-462.

15. Kidney J, Dominguez M, Taylor PM, Rose M, Chung KF, Barnes PJ. Immunomodulation by theophylline in asthma: demonstration by withdrawal of therapy. Am J Respir Crit Care Med 1995; 151: 1907-1914.

16. Finnerty JP, Lee C, Wilson S, Madden J, Djukanovic R, Holgate ST. Effects of theophylline on inflammatory cells and cytokines in asthmatic subjects: a placebo-controlled parallel group study. Eur Respir J 1996; 9: 1672-1677.

17. Sullivan P, Bekir S, Jaffar Z, Page C, Jeffery P, Costello J. Anti-inflammatory effects of low-dose oral theophylline in atopic asthma. Lancet 1994; 343: 1006-1008.

18. Gristwood RW, Llupia J, Fernandez AG, Berga P. Effects of theophylline compared with prednisolone on late phase airway leukocyte infiltration in guinea pigs. Int Arch Allergy Appl Immunol 1991; 94: 293-294.

19. Lagente V, Pruniaux M-P, Junien J-L, Moodley I. Modulation of cytokine-induced eosinophil infiltration by phosphodiesterase inhibitors. Am J Respir Crit Care Med 1995; 151: 1720-1724.

20. Kraft M, Torvik JA, Trudeau JB, Wenzel SE, Martin RJ. Theophylline: potential antiinflammatory effects in nocturnal asthma. J Allergy Clin Immunol 1996; 97: 1242 1246.

21. Hatzelmann A, Tenor H, Schudt C. Differential effects of non-selective and selective phosphodiesterase inhibitors on human eosinophil functions. Br J Pharmacol 1995; 114: 821-831.

22. Berends C, Dijkhuizen B, de Monchy JGR, Dubois AEJ, Gerritsen J, Kauffman HF. Inhibition of PAF-induced expression of CD11b and shedding of L-selectin on human neutrophils and eosinophils by the type IV selective PDE inhibitor, rolipram. Eur Respir J 1997; 10: 1000-1007.

23. Berends C, Hoekstra MO, Dijkhuizen B, de Monchy JGR, Gerritsen J, Kauffman HF. Expression of CD35 (CR1) and CD11b (CR3) on circulating neutrophils and eosinophils from allergic asthmatic children. Clin Exp Allergy 1993; 23: 926-933.

24. de Grooth BG, Terstappen LWMM, Puppels GJ, Greve J. Light scattering polarization, a new parameter in flow cytometry. Cytometry 1987; 8: 539-544.

25. Currie MS, Rao KM, Padmanabhan J, Jones A, Crawford J, Cohen HJ. Stimulus-specific effects of pentoxifylline on neutrophil CR3 expression, degranulation, and superoxide production. J Leukoc Biol 1990; 47: 244-250.

26. Sagara H, Fukuda T, Okada T, Ishikawa A, Makino S. Theophylline at therapeutic concentration suppresses PAFinduced upregulation of Mac-1 on human eosinophils. Clin Exp Allergy 1996; 26: Suppl. 2, 16-21.

27. Yukawa T, Kroegel C, Chanez P, et al. Effect of theophylline and adenosine on eosinophil function. Am Rev Respir Dis 1989; 140: 327-333.

28. Nielson CP, Vestal RE, Sturm RJ, Heaslip R. Effects of selective phosphodiesterase inhibitors on the polymorphonuclear leukocyte respiratory burst. J Allergy Clin Immunol 1990; 86: 801-808.

29. Chan SC, Reifsnyder D, Beavo JA, Hanifin JM. Immunochemical characterization of the distinct monocyte cyclic AMP-phosphodiesterase from patients with atopic dermatitis. J Allergy Clin Immunol 1993; 91: 1179-1188.

30. Bachelet M, Vincent D, Havet N, et al. Reduced responsiveness of adenylate cyclase in alveolar macrophages from patients with asthma. J Allergy Clin Immunol 1991; 88: 322-328. 